\title{
Experimental Demonstration of a $10 \mathrm{~Gb} / \mathrm{s}$ 16-QAM SCM WDM PON with Bandwidth-limited RSOA and IM/DD Transceivers
}

\author{
Jonathan M. Buset ${ }^{(1)}$, Ziad A. El-Sahn ${ }^{(1,2)}$, and David V. Plant ${ }^{(1)}$ \\ (1) Photonic Systems Group, Department of Electrical and Computer Engineering, McGill University, \\ Montréal, QC, H3A 2A7, Canada, (jonathan.buset@mail.mcgill.ca) \\ ${ }^{(2)}$ Electrical Engineering Department, Alexandria University, Alexandria, 21544, Egypt.
}

\begin{abstract}
We demonstrate $10 \mathrm{~Gb} / \mathrm{s} / \lambda$ full-duplex transmission over a $20 \mathrm{~km}$ single feeder WDM PON. DSP enables spectrally efficient 16-QAM modulation and RF subcarrier multiplexing (SCM) for both uplink and downlink channels using low cost optoelectronics. BER performance below the RS $(255,223)$ FEC threshold is verified.
\end{abstract}

\section{Introduction}

The bandwidth capacities of access networks must evolve as consumer appetites for high bandwidth services continue to grow. Wavelength-division multiplexed passive optical networks (WDM PONs) that build on existing transport network technologies are expected to provide the flexibility and capacity to meet future needs upwards of $10 \mathrm{~Gb} / \mathrm{s}$ per user. ${ }^{1}$ Colourless optical network units (ONUs) are essential to reduce inventory complexity for the network operation. The operation of bandwidth-limited reflective semiconductor optical amplifier (RSOA)-based ONUs at $10 \mathrm{~Gb} / \mathrm{s}$ or greater data rates has been demonstrated using a variety of electrical ${ }^{2,3}$ and optical ${ }^{4-6}$ equalization techniques. Advancements in digital signal processing (DSP) techniques have enabled spectrally efficient next generation (NG)-PON solutions. ${ }^{7-10}$

In this paper we demonstrate the feasibility of transmitting $10 \mathrm{~Gb} / \mathrm{s}$ 16-QAM data streams from a bandwidth-limited RSOA and economical intensity modulation (IM)/direct detection (DD) optoelectronics. The RSOA's $2.2 \mathrm{GHz}$ modulation bandwidth is spectrally pre-compensated in DSP which facilitates transmitting spectrally efficient high-order modulation formats. ${ }^{10}$ Offset optical filtering is used at the optical line terminal (OLT) to further enhance the chirped upstream signal. RF subcarrier multiplexing (SCM) reduces the impact of inter-channel crosstalk and Rayleigh backscattering (RB) due to bidirectional transmission. We demonstrate operation over a $20 \mathrm{~km}$ WDM PON and characterize the system in terms of bit error ratio (BER) performance. Scenarios with remote continuous-wave (CW) seeding and wavelength re-use of the modulated downlink signal are both presented. To the best of our knowledge this is the first demonstration of a single carrier $10 \mathrm{~Gb} / \mathrm{s}$ SCM WDM PON using a commercial RSOA with wavelength re-use and IM/DD 16-QAM transmission for both the uplink and downlink directions.

\section{PON Physical Architecture}

Fig. 1 illustrates the single feeder architecture used in this proof of concept experiment. The OLT transmitter consists of a Micram VEGA digital-to-analog converter (DAC) with 6 bit precision driving an electro-absorption modulated laser (EML) centred at $1549.41 \mathrm{~nm}$. Following the EML, a booster erbiumdoped fiber amplifier (EDFA) and variable optical attenuator (VOA) control the launch power $P_{O L T_{-} T x}$, as measured at the circulator output. The downlink extinction ratio (ER) is set with a bias-tee to achieve a minimum BER. The optical distribution network (ODN) comprises a $20.35 \mathrm{~km}$ feeder of standard single mode fiber (SMF-28e+), a $100 \mathrm{GHz}$ arrayed waveguide grating (AWG) and a $1.5 \mathrm{~km}$ distribution drop fiber (DDF).

At the ONU, 70\% of the downstream signal is tapped off for detection by the receiver comprised of a p-i-n photoreceiver, a RF amplifier, and a real-time oscilloscope performing 8 bit precision analog-todigital conversion (ADC). The remaining $30 \%$ of the downstream signal seeds a RSOA with peak gain from $1530 \mathrm{~nm}$ to $1570 \mathrm{~nm}$ and $2.2 \mathrm{GHz}$ modulation bandwidth. The RSOA uplink transmitter is directly modulated at $4 \mathrm{~V}_{\mathrm{p}-\mathrm{p}}$, biased at $80 \mathrm{~mA}$, and driven by a second VEGA DAC. At the OLT, an EDFA preamplifies the upstream signal prior to detection and a VOA sets the input power to the $\mathrm{p}$-i-n. In a realworld deployment the EDFA cost would be shared 


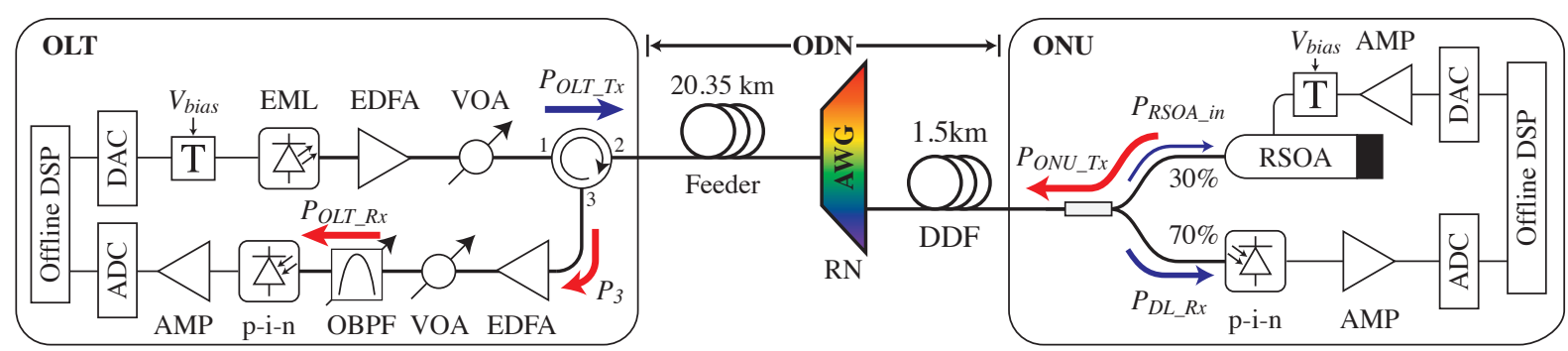

Figure 1. Physical architecture of the SCM WDM PON. The ODN losses are $9.19 \mathrm{~dB}$. The optimized 70/30 ONU coupler balances the power budgets. AMP: RF amplifier, AWG: arrayed waveguide grating, EDFA: erbium-doped fiber amplifier, EML: electro-absorption modulated laser (CyOptics E4560), OBPF: $0.3 \mathrm{~nm}$ optical band-pass filter (JDSU VCF050), p-i-n: 10 GHz photoreceiver (DSC-R402), RN: remote node, RSOA: reflective semiconductor optical amplifier (CIP SOA-RL-OEC-1550), T: RF bias-tee, VOA: variable optical attenuator.

among the PON's user base. An optical band pass filter (OBPF) is offset from the carrier wavelength by $-0.18 \mathrm{~nm}$ to optically enhance the received signal while also emulating a second AWG for wavelength demultiplexing. The OLT receiver is similar to that of the ONU consisting of a p-i-n photoreceiver, a RF amplifier, and an ADC to capture the received signal.

\section{DSP Enabled $10 \mathrm{~Gb} / \mathrm{s}$ 16-QAM}

Fig. 2 outlines the general flow of the transmitter and receiver software stacks. ${ }^{10}$ At the transmitters, $2^{23}-1$ and $2^{31}-1$ length pseudo-random bit sequences (PRBSs) are generated offline for the downlink and uplink, respectively. A short $\sim 1 \mathrm{kSymbol}$ preamble is added to the beginning of each sequence. After 16-QAM symbol mapping the sequences are truncated to $512 \mathrm{kSymbol}$ due to DAC memory limitations. A square-root raised cosine (SRRC) filter of order 128 is then applied to increase the spectral efficiency and reduce the effects of intersymbol interference (ISI). Prior to transmission, a finite impulse response (FIR) filter is applied to the signal to pre-compensate for each channel's bandwidth limiting component. This flattens the power spectrum over the band of interest enabling transmission and reception of 16-QAM electrical signals with low-cost optoelectronics. The DACs then drive the optical transmitters with the quantized signals.

The data captured at $40 \mathrm{GSa} / \mathrm{s}$ by the $\mathrm{ADC}$ receiver is downsampled, $\mathrm{RF}$ down-converted, and a matched SRRC filter (order 128) is applied to eliminate ISI and remove out-of-band noise. After the timing synchronization and QAM decision blocks, the preamble symbols are used to remove the phase-locked loop (PLL) phase ambiguity. Post-compensation can optionally be performed using a decision feedback equalizer (DFE) with symbol spaced taps. The DFE taps are initially trained using the preamble symbols

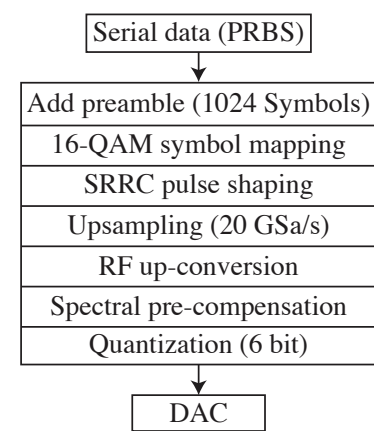

(a)

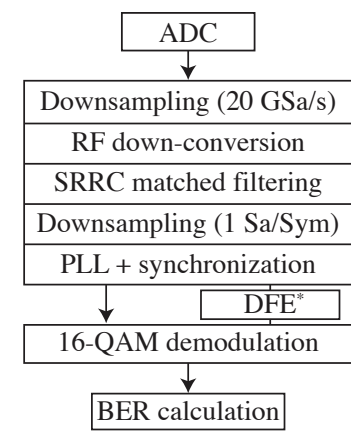

(b)
Figure 2. Block diagrams of the (a) transmitter and (b) receiver DSP stacks used for offline processing of the downlink and uplink signals. ${ }^{*}$ Note that DFE post-compensation is optional.

and then dynamically adjusted using the least-mean square (LMS) algorithm. The signal is then demodulated for BER calculation using approximately $3 \times 10^{6}$ bit of data.

\section{Experimental Results}

The 2.5 Gbaud 16-QAM uplink signal is up-converted on a $2.97 \mathrm{GHz}$ RF subcarrier. The SRRC roll off factor $\alpha_{U L}=1.0$ results in a first null bandwidth electrical bandwidth of $5 \mathrm{GHz}$. A 64th order FIR filter pre-compensates for the RSOA bandwidth roll off prior to quantization. To characterize the performance of the upstream transmission, the OLT launch power $P_{O L T_{-} T x}$ was fixed at $5 \mathrm{dBm}$. This corresponds to an RSOA input power $P_{R S O A_{-} i n}=-10.7 \mathrm{dBm}$, which is greater than the RSOA's saturation power of $-17 \mathrm{dBm}$. The ONU gain is $\sim 12.9 \mathrm{~dB}$ resulting in an upstream $P_{O N U_{-} T x}=2.2 \mathrm{dBm}$. After transmission through the ODN, the received power at port 3 of the circulator is measured to be $P_{3}=-7.6 \mathrm{dBm}$.

In Fig. 3 we present the BER performance of the $10 \mathrm{~Gb} / \mathrm{s}$ upstream transmission with both remote $\mathrm{CW}$ seed and symmetric transmission with wavelength reuse from a portion of the modulated downlink sig- 


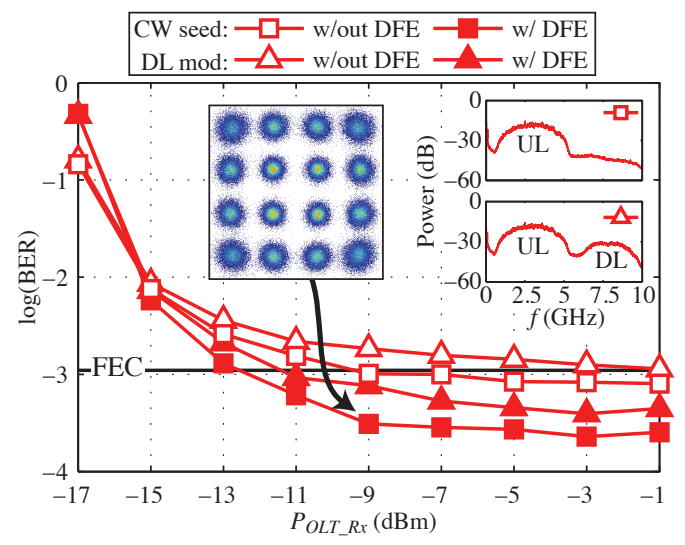

Figure 3. Uplink BER performance of the upstream after transmission over a $20 \mathrm{~km}$ PON. Equalized constellation and normalized power spectra at $-9 \mathrm{dBm}$ are inset. A FEC threshold of BER $1.1 \times 10^{-3}$ is assumed for $\operatorname{RS}(255,223)$. Note the presence of the reflected downlink channel in the symmetric transmission case.

nal. We assume standard Reed-Solomon (RS) forward error correction (FEC) codes with $12.5 \%$ overhead.

With remote $\mathrm{CW}$ seeding, the uplink can achieve a BER below the $\operatorname{RS}(255,223)$ FEC threshold at $-9 \mathrm{dBm}$ of received power. In symmetric operation a post-compensation DFE with 8 forward and 1 backward symbol spaced taps improves the uplink receiver sensitivity, requiring $-11 \mathrm{dBm}$ to operate below the FEC threshold. Separating the channels with SCM reduces the impact of inter-channel crosstalk, and in terms of power penalty the difference between $\mathrm{CW}$ seeding and symmetric transmission is less than $2 \mathrm{~dB}$.

The 2.5 Gbaud 16-QAM downlink channel is upconverted on a $7.97 \mathrm{GHz}$ RF subcarrier with a SRRC roll off factor $\alpha_{D L}=0.5$. The smaller $\alpha_{D L}$ reduces the first null electrical bandwidth to $3.75 \mathrm{GHz}$ ensuring spectral separation from the uplink channel. A 16th order FIR filter pre-compensates for the $10 \mathrm{GHz}$ p-i-n bandwidth roll off. In Fig. 4 we confirm the downlink channel operation by characterizing its BER performance as a function of $P_{O L T} T x$. The downlink BER quickly reaches below the FEC threshold for OLT launch powers greater than $1 \mathrm{dBm}$. This corresponds to a received power sensitivity of $\sim-11 \mathrm{dBm}$.

\section{Discussion}

The proposed $10 \mathrm{~Gb} / \mathrm{s}$ 16-QAM SCM WDM PON solution builds on a previous demonstration that used 5 Gbaud QPSK. ${ }^{10}$ Using a simple wavelength re-use scheme and commodity IM/DD transceivers at both the OLT and ONU simplifies the optoelectronic requirements, while DSP enables pulse shaped higher order modulation formats to increase the RF

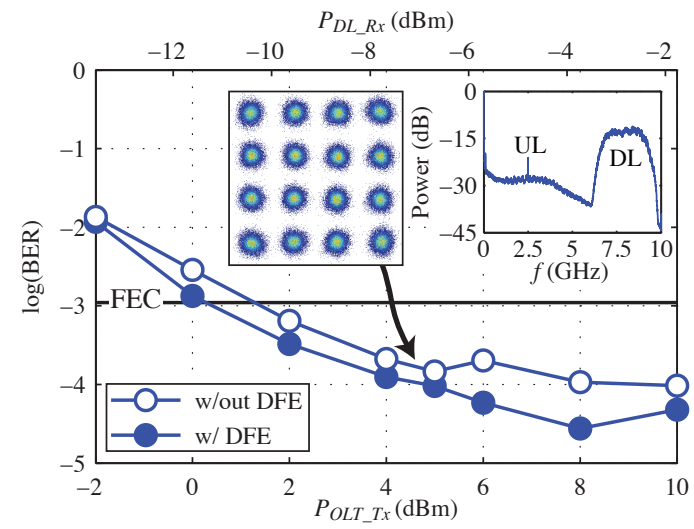

Figure 4. Downlink channel BER after transmission over a $20 \mathrm{~km}$ PON. The received power is also presented for completeness. The optional ONU DFE consists of 2 forward and 1 backward symbol spaced taps. Equalized constellation and normalized power spectrum at $P_{O L T \_T x}=5 \mathrm{dBm}$ are inset.

spectral efficiency and provides robust resistance to inter-channel crosstalk by separating the uplink and downlink channels. Furthermore, moving the uplink channel away from the baseband drastically reduces the impact of low frequency RB beat noise ${ }^{11}$ during the DSP down-conversion and matched filtering stages.

\section{Conclusion}

In this paper we demonstrate a new single feeder $10 \mathrm{~Gb} / \mathrm{s}$ 16-QAM SCM WDM PON architecture that maximizes the performance of low cost IM/DD optoelectronics using DSP techniques. We verify the system's performance over a single $20 \mathrm{~km}$ feeder with both remote $\mathrm{CW}$ seeding and full-duplex transmission, achieving BERs below the RS $(255,223)$ FEC threshold at $5 \mathrm{dBm}$ of OLT launch power.

\section{References}

[1] J.-I. Kani, IEEE J. Sel. Topics Quantum Electron. 16, $1290-1297$ (2010).

[2] K. Y. Cho et al., IEEE Photon. Technol. Lett. 20, 15331535 (2008).

[3] J. Prat, Proc. ECOC, Th.10.B.3 (2010).

[4] I. Papagiannakis et al., IEEE Photon. Technol. Lett. 20, 2168-2170 (2008).

[5] H. Kim, IEEE Photon. Technol. Lett. 22, 1379-1381 (2010).

[6] M. Presi et al., Opt. Express 20, B507-B512 (2012).

[7] N. Cvijetic, J. Lightw. Technol. 30, 384-398 (2012).

[8] K. Y. Cho et al., Opt. Express 20, 15,353-15,358 (2012).

[9] D. Lavery et al., Opt. Express 18, 25,855-25,860 (2010).

[10] J. M. Buset et al., Proc. OFC/NFOEC, NTh4F (2013).

[11] A. Chiuchiarelli et al., IEEE Photon. Technol. Lett. 22, 85-87 (2010). 